Ann. Zootech., Ig68, 17 (3), 245-256.

\title{
TRAITE A LA MACHINE DES BREBIS. CONSÉQUENCES DE LA SUPPRESSION DE L'ÉGOUTTAGE MANUEL EN FONCTION DES CARACTÉRISTIQUES DE TRAITE
}

\author{
G. RICORDEAU, J. LABUSSIÈRE \\ Station centrale de Génétique animale, \\ et Laboratoire de Physiologie de la Lactation \\ Centre national de Recherches zootechniques, 78 -Jouy-en-Josas \\ Institut national de la Recherche agronomique
}

SOMMAIRE

Cette étude a pour but d'estimer les variations de production consécutives à la suppression de l'égouttage manuel (traite de finition effectuée à la main après enlèvement des gobelets), en fonction des quantités de lait obtenues au cours des différentes phases de traite.

Deux essais ont été réalisés : l'un en ferme sur 202 brebis Lacaune (dont Ior témoins) traites après 38 jours d'allaitement ; l'autre en station sur 38 Préalbes du Sud et $5 \mathrm{I}$ croisées traites depuis la mise bas.

La suppression de l'égouttage manuel a été effectuée pendant 20 jours, à partir du $z^{\mathbf{e}}$ mois de lactation. Elle entraîne une perte de i $8 \mathrm{ml}$ ( 6 p. 100) supérieure au volume de l'égouttage obtenu dans le lot témoin $(93 \mathrm{mI})$.

Nous avons montré que la chute globale de production dépend de facon significative du volume initial d'égouttage, même après correction de l'influence du niveau de production $(r=0,34)$. En revanche, la différence entre les quantités de lait obtenues à la machine avant et après suppression de l'égouttage varie en sens inverse du volume d'égouttage et semble plus faible pour les brebis cédant la plus grande partie de leur lait au cours des 30 premières secondes de traite.

\section{IN'TRODUC'TION}

La traite à la machine des brebis comprend habituellement plusieurs opérations. Un premier trayeur pose les gobelets sans préparation de la mamelle, puis, lorsque le débit du lait diminue ou a cessé, il effectue l'égouttage-machine en massant la glande et enlève les gobelets. Quelques minutes après, un deuxième trayeur effectue la traite de finition à la main en soubattant plus ou moins énergiquement : c'est l'égouttage manuel ou " repasse ". Cette dernière opération nécessite de bons trayeurs 
et réduit considérablement le nombre de brebis traites par ouvrier et par heure ; is est donc souhaitable de chercher à la supprimer. Dans une étude précédente (RIcoRDEAU, MARTINet et Denamur, I963) nous avons mesuré de façon globale la chute de production consécutive à la suppression de l'égouttage. L'objet de cette étude est d'estimer de façon plus précise les conséquences de cette suppression, compte tenu des différences individuelles dans le niveau de production, la quantité d'égonttage manuel et la vitesse de traite.

\section{MATÉRIEL, E'T TECHNIQUES}

Deux essais ont été réalisés, l'un dans une ferme de la région de Roquefort, l'autre au domaine de Brouessy. Dans les deux cas, on a utilisé le protocole suivant :

période I : ro jours avec contrôle de la traite-machine et de l'égouttage manuel.

période $2: 20$ jours sans égouttage manuel.

période 3 : ro jours avec rétablissement de l'égouttage manuel.

la période 4 (fin de la lactation avec égouttage) concerne uniquement l'essai en ferme sur Lrebis Lacaune.

r. Dans l'essai en ferme, on utilise 202 antenaises et adultes de race Lacaune. L'allaitement dure 38 jours en moyenne. La période $\mathrm{x}$ commence 7 jours après la mise à la traite, soit le $45^{\mathrm{e}}$ jour de lactation. L'égouttage manuel est supprimé sur Ior brebis (lot expérimental) les autres constituant le témoin. Les contrôles volumétriques de production sont effectués sur les 2 traites quotidiennes, tous les deux jours pendant les périodes I, 2 et 3 , tous les mois après le $85^{\mathrm{e}}$ jour de lactation.

Ce troupeau est soumis à la traite mécanique pour la première fois dans une installation de type "Casse " suivant la technique préconisée par Bosc (1962).

2. L'essai en station porte sur deux années. En 1963 , on utilise 89 brebis en $2^{\mathrm{e}}$ lactation dont 38 de race Préalpes du Sud et $5 \mathrm{I} \mathrm{F}_{\mathbf{1}}$ issues du croisement Frison $\times$ Préalpes. Ces brebis sont traites depuis la mise bas. La période I commence le $5^{\circ}$ jour de lactation. Le contrôle laitier volumétrique est effectué quotidiennement sur les 2 traites, pendant toute la lactation. La cinétique de la descente de lait est contrôlée sur une période de 2 jours (soit 4 traites consécutives) ì la fin de la période $\mathbf{I}$ ( 59 et $60^{\mathrm{e}}$ jour de lactation) et de la période 2 ( 79 et $80^{\mathrm{e}}$ jour de lactation) : les mesures sont effectuées toutes les I5 secondes suivant une technique décrite antérieurement (Ricordeau, Martinet, Denamur, 1963 ).

En I 964, l'essai est répété sur 40 brebis croisées. Les enregistrements de descente du lait sont effectués uniquement à la fin de la période $z$ avec un suiveur de niveau (LARUSSİ̀re et MarTineT, 1964), permettant de mesurer les volumes de lait toutes les 4 secondes.

En I963 et ig64, il n'y a pas eu de lot témoin dans l'essai en station.

\section{Definitions.}

M : quantité de lait obtenue à la machine, y compris l'égouttage-machine.

$\mathrm{E}$ : quantité de lait obtenue à l'égouttage manuel.

$\mathrm{T}$ : volume total de la traite.

E \%: volume de l'égouttage manuel en p. soo du volume de lait obtenu à la machine.

Les indices correspondent aux 3 premières périodes de contrôles.

$\mathrm{D}_{i}=$ différence entre les quantités totales de lait obtenues entre les périodes 1 et $\mathbf{2}$, soit $\mathrm{T}_{1}-\mathrm{M}_{2}$.

$d_{i}=$ différence entre les quantités de lait-machine obtenues entre les périodes I et 2 , soit $\mathrm{M}_{1}-\mathrm{M}_{2}$.

$\mathrm{D}_{i}$ corrigée $=$ différence entre la quantité totale moyenne des périodes I et 3 et la quantité obtenue en période 2 , soit [moyenne $\mathrm{T}_{1}$ et $\mathrm{T}_{3}-\mathrm{M}_{2}$ ].

La vitesse de traite $(\% 30 \mathrm{~s})$ est définie par la quantité de lait obtenue au cours des $3^{\circ}$ premières secondes de traite en p. Ioo de la quantité obtenue à la traite-machine proprement dite (c'est-à-dire non compris le lait de l'égouttage-machine obtenu après intervention du trayeur). 
4. Caractéristiques de la machine à traire.

Dans les 2 essais, on utilise une machine Alfa-Laral : Vide de $33 \mathrm{~cm}$ de mercure, vitesse de pul. sation de $180 / \mathrm{mn}$, rapport $\mathrm{I} / \mathrm{I}$.

\section{RÉSUL'TA'TS}

\section{I. - Comparaison entre les périodes 1 et 2}

- Essai en ferme (Lacaune).

- Pendant les ro jours de la période I, le volume moyen de l'égouttage est de $97 \mathrm{ml}\left({ }^{1}\right)$, soit $14,3 \mathrm{p}$. Ioo du volume de lait trait à la machine. La figure I donne une idée de la variabilité observée entre animaux dans un troupeau ordinaire : 20 à $280 \mathrm{ml}, 3$ à $5^{2} \mathrm{p}$. IOO.
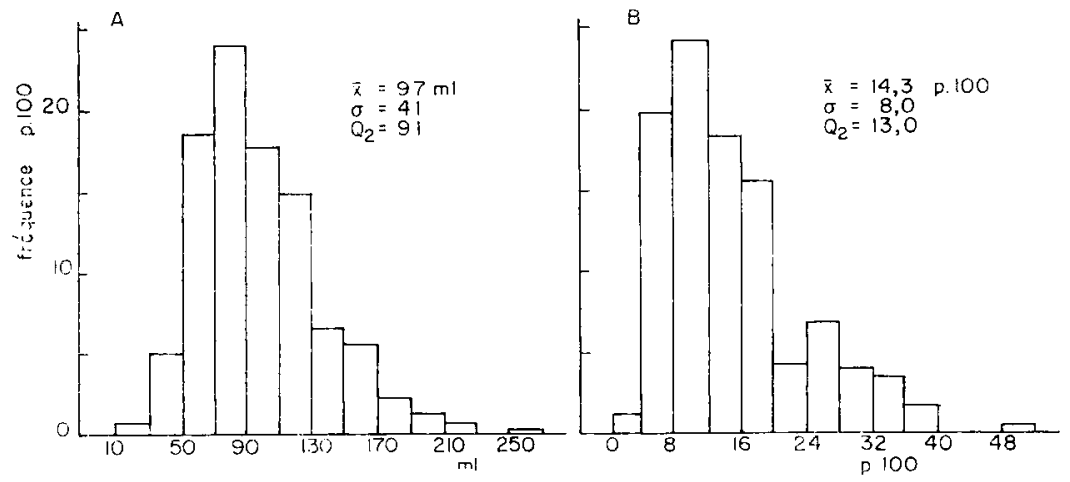

FIs. 1. - Imprertance de l'égonitage manuel calculé sur 10 traites au cours de la périodé n' (202 brebis Lacaunes de l'essai en ferme)

A - Quaniliés absolues $\mathrm{cn}$ ml de lait par jour

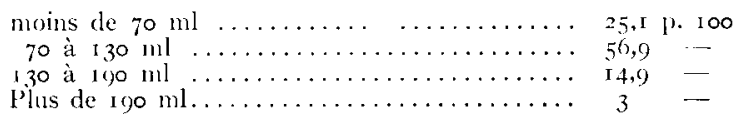

$B-$ En p. 100 du volume de lail oblenu à la machine ( $\mathrm{E} \%$ )

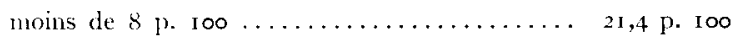

8 à 16 p. $100 \ldots \ldots \ldots \ldots \ldots \ldots \ldots \ldots \ldots, 42,7 \quad \ldots$

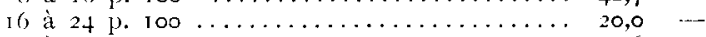

24 à 32 p. $100 \ldots \ldots \ldots \ldots \ldots \ldots \ldots \ldots \ldots$ 10,6 $\ldots$

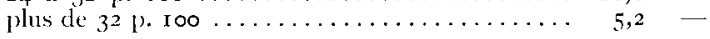

$x=$ moyenne $; \quad \sigma=$ écart-type $; \quad Q_{2}=$ médiane

- Pendant la période 2, la comparaison entre le lot témoin et le lot expéri. mental (tabl. I, fig. 2), indique que l'absence d'égouttage entraîne une perte jour. nalière de I $8 \mathrm{ml}$ ( $107 \mathrm{ml}$ les ro premiers jours et $\mathrm{I} 30 \mathrm{ml}$ les ro jours suivants) qui

(1) Dans le lot témoin, le classement des brebis en 2 groupes (l'un dont le niveau d'égouttage en période I est supérieur à $90 \mathrm{ml}$ par jour, l'autre dont le niveau est inférieur à $90 \mathrm{ml}$ ) montre que le volume d'égouttage reste constant pour les brebis à faible niveau $(68,69,65$ et $6+\mathrm{ml}$ au cours des périodes $\mathrm{I}, 2,3$ et + ) mais diminue nour les brebis à haut niveau (respectivement : $128,11_{7}, 104,83 \mathrm{ml}$ ). 


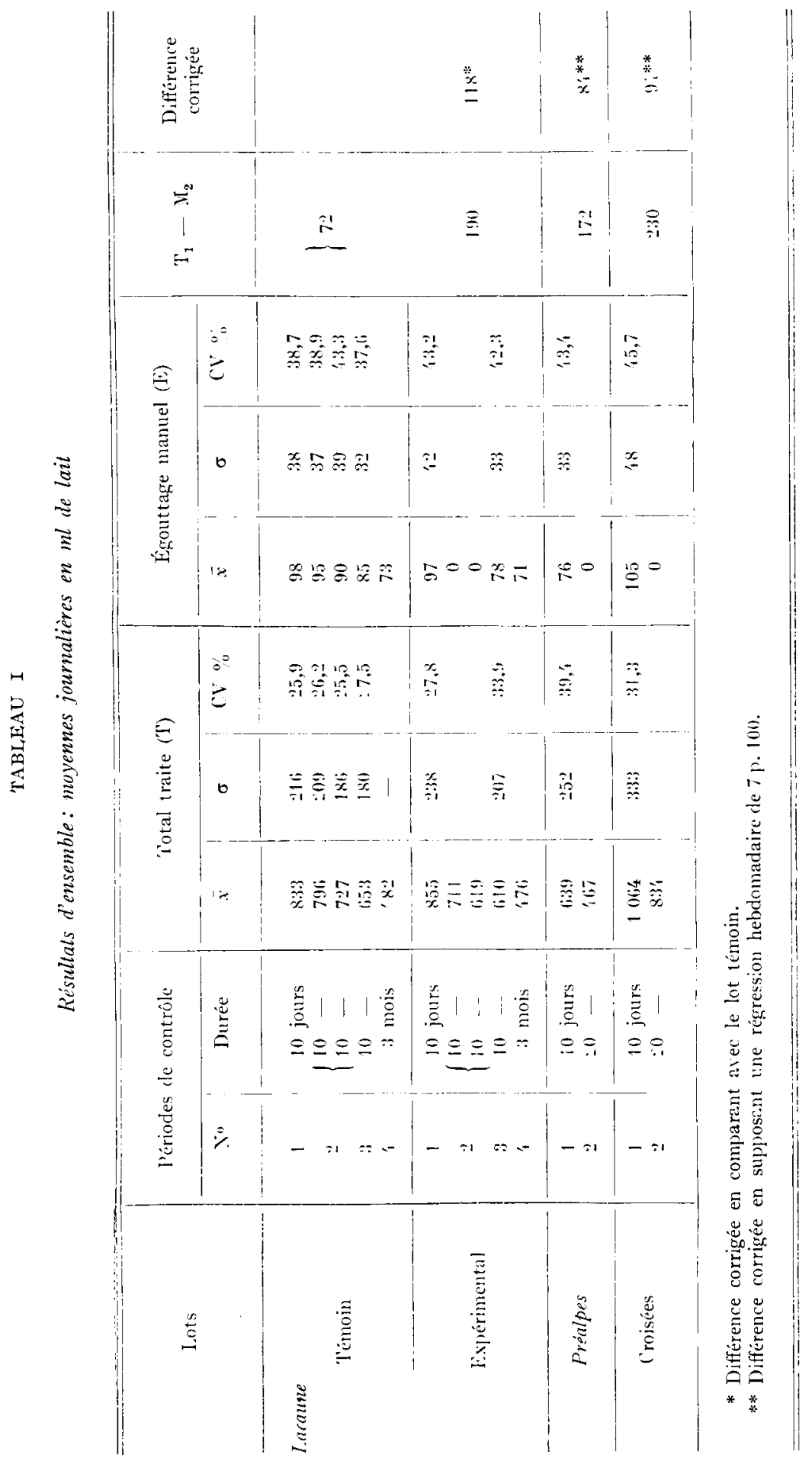


représente 16 p. Ioo de la production totale dı lot témoin. Cette perte est supérieure de $25 \mathrm{ml}$ au volume d'égouttage, obtenu dans le lot témoin.

- Lors de la $3^{\mathrm{e}}$ période, après rétablissement de l'égouttage manuel, la différence de production n'est plus que de $65 \mathrm{ml}$ de lait ; elle devient pratiquement négligeable I mois après rétablissement de l'égouttage manuel (période 4, tabl. I et fig. 2).

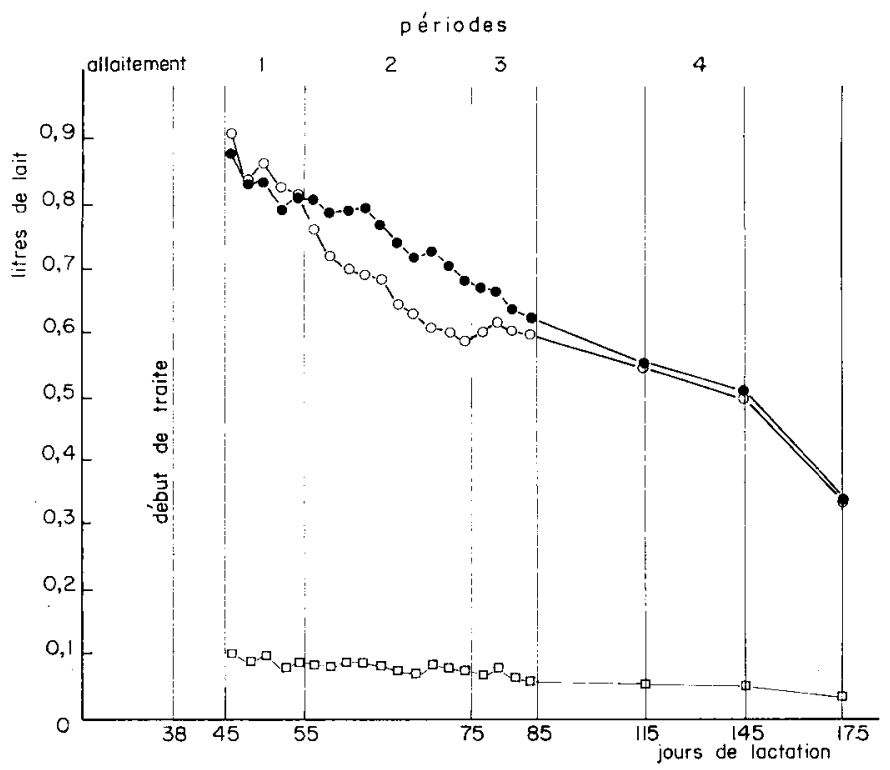

FIG. 2. - Évolution de la production laitière du lot égoulté et du lot non égoutté à la main dans l'essai en lerme (brebis de race Lacaune)

- Lot témoin

- Lot expérimental, sans égouttage manuel pendant la période 2

口 Quantité de lait obtenue à l'égouttage manuel dans le lot témoin

- Essai en station (Préalpes et croisées).

Entre les périodes $\mathrm{I}$ et 2 , la chute de production est de $\mathrm{I} 72 \mathrm{ml}$ de lait pour les Préalpes et de $230 \mathrm{ml}$ pour les croisées. En 1'absence de témoin, on doit tenir compte de la décroissance de production laitière en fonction du stade de lactation ( $7 \mathrm{p}$. Ioo en moyenne par semaine; RICORDEAU et DENAMUR, I962). Après correction, cette perte représente respectivement 84 et $94 \mathrm{ml}$ de lait par jour, soit I4 et II p. Ioo de la production théorique que l'on pouvait espérer au cours de la période 2 (tabl. I).

\section{II. - Infuence du niveau de production obtenu à la machine et à l'égouttage manuel}

La chute de production laitière enregistrée entre les périodes I et 2 varie de façon importante d'une brebis à l'autre. Pour essayer d'expliquer ces variations individuelles, nous avons effectué une analyse statistique portant sur 2 différences.

I. La différence $D_{i}$ exprime la chute totale de production entre les périodes I et 2 . Elle est en corrélation positive avec le volume d'égouttage $\mathrm{F}_{\mathbf{1}}$ et avec le niveau 
de production $\mathrm{T}_{1}$ (tabl. 2), mais la régression totale ou partielle de $\mathrm{D}_{i}$ sur $\mathrm{E}_{1}$ est inférieure à l'unité $(b=0,85)$. Aussi ne peut-on espérer réđuire de façon sensible la chute de production consécutive à la suppression de l'égouttage en éliminant les brebis cédant habituellement beaucoup de lait lors de cet égouttage. Au contraire, le niveau de production obtenu sans égouttage en période 2 (c'est-à-dire $\mathrm{M}_{2}$ ) est indépendant du volume initial d'égouttage et dépend étroitement de la quantité totale et de la quantité de lait-machine obtenue en période I (tabl. 2).

\section{TABLEAU 2}

Corrélations entre paramètres de production: moyennes concernant 190 brebis des 3 lots soumis à la suppression de l'égouttage

\begin{tabular}{|c|c|c|c|c|}
\hline Variables & $\lambda i_{1}$ & $\mathrm{E}_{1}$ & $\dddot{i}_{1}$ & $\mathrm{M}_{2}$ \\
\hline $\mathrm{E}_{1}$ & $-0,11$ & & & \\
\hline$i_{1}$ & $+0,99^{* *}$ & +0.07 & & \\
\hline $\mathrm{M}_{2}$ & $+0,95^{* *}$ & $-0,06$ & $+0,9^{\prime * *}$ & \\
\hline $\mathrm{D} i=\mathrm{T}_{1}-\mathrm{\lambda}_{2}$ & $+0,38 *$ & $+0,30^{* *}$ & $+0,13^{* *}$ & $+0,12 * *$ \\
\hline $\mathrm{I}_{i}$ corrige $\left(\mathrm{I}_{1 \cdot 3}-\mathrm{Mi}_{2}\right)$ & $+0,17$ & $+0,3, * *$ & $\overline{+0,14}$ & $+0,02$ \\
\hline$\% F_{1}$ & $-0,6^{\prime} * *$ & $+0,75^{* *}$ & $-0,51 * *$ & $-0,60^{* *}$ \\
\hline
\end{tabular}

2. I, a variable $\mathrm{T}_{1}$ étant la somme de $\mathrm{E}_{1}$ et de $\mathrm{M}_{1}$, il est assez difficile de séparer l'influence respective de ces 2 facteurs. Pour traduire la variation de production consécutive à la suppression de l'égouttage, nous prendrons donc comme paramètre la différence $d_{i}=\mathrm{M}_{1}-\mathrm{M}_{2}$.

- Essai en ferme.

L'analyse de la variance des $d_{i}$ (lot témoin avec lot expérimental) révèle une influence significative du niveau de lait-machine, mais pas du niveau d'égouttage (tab1. 3). Cependant, les résultats moyens du tableau $4 a$ semblent indiquer que l'influence du niveau d'égouttage pourrait ne pas être la même pour le lot expérimental et pour le lot témoin : l'effet lot est d'ailleurs significatif.

- Essai en station.

L'analyse de la variance des $d_{i}$ (tabl. 3) fait apparaître une influence significative du niveau de lait-machine et du niveau d'égouttage. Cependant, si $d_{i}$ varie dans le même sens que $\mathrm{M}, d_{i}$ diminue lorsque $\mathrm{E}$ augmente. Cela signifie que, pour un même niveau de production, les brebis donnant habituellement beaucoup de lait à l'égouttage perdent moins de lait à la machine lorsqu'on supprime la finition à la main (tabl. 4). 
TRAITE A IA MACHINE DES BREBIS

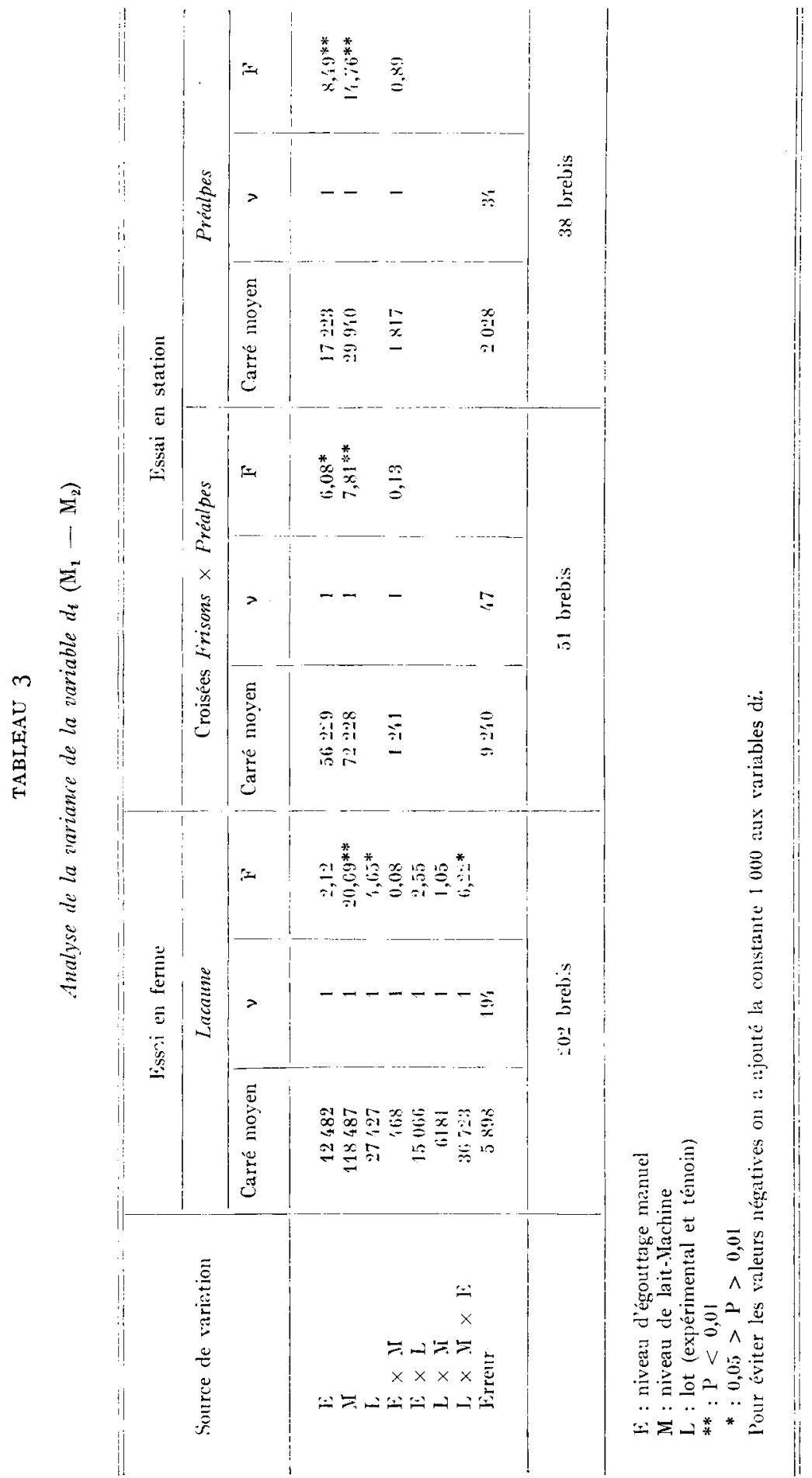


Ce résultat s'observe dans les 2 essais, sauf pour le lot témoin Lacaune, ce qui semble traduire l'existence d'un phénomène de compensation. On peut également constater la similitude des réponses entre les Lacaune de Roquefort et les Préalpes de Brouessy (tab1. $4 a$ ). La différence $d_{i}$ est plus élevée pour les croisées Frison $\times$ Préalpes, mais cela tient au niveau de production.

\section{TABI,EAU 4}

Influence du niveau d'égouttage manuel et du niveau de production obtemu à la machine

a) Différences $d_{i}$ en $m l$ de lait par jour

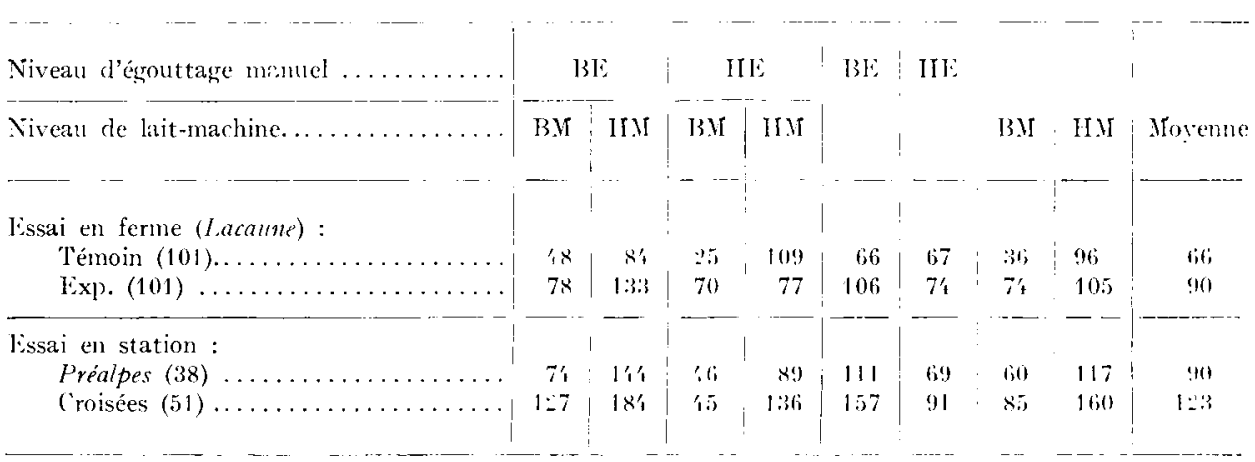

b) vitesse de traite $(\% 30 \mathrm{~s})$ : essai en station

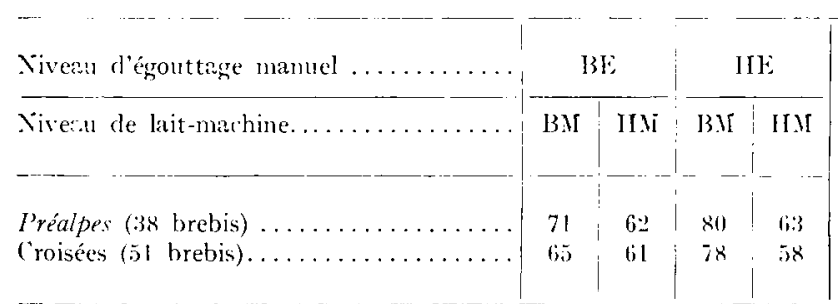

Chaque lot a été divisé en z moitiés, hatut et bus niveatr:

\section{III. - Caractéristiques de traite}

Il est intéressant de savoir si les brebis qui s'adaptent bien à la suppression de l'égouttage manuel sont celles qui se laissent bien traire à la machine. L'aptitude à la traite est un critère qui n'est pas encore défini avec précision mais les études récentes sur la cinétique de la descente du lait ont permis de distinguer 2 catégories de brebis : celles qui donnent leur lait en I fois (brebis à $I$ pic) et celles qui le donnent en $2 \mathrm{fc}$ is (brebis à 2 pics). Pour traduire ces différences, nous avons calculé, pour 
chaque brebis, la proportion de lait obtenue au cours des 30 premières secondes de traite en fonction de la quantité obtenue à la traite-machine proprement dite. I'après les observations de LABUSSIÈRE et MARTINET (I 964), on peut considérer que le lait des 30 secondes représente approximativement le lait citernal. Les résultats obtenus sont résumés dans le tableau $5:$ la différence $d_{i}$ est plus faible pour les brebis qui cèdent la plus grande partie de leur lait en đébut de traite. Ce résultat a été confirmé en I 964 sur les brebis contrôlées avec le suiveur de niveau : $d_{i}$ est en moyenne de $60 \mathrm{ml}$ pour les brebis classées à I pic et de $195 \mathrm{ml}$ pour les brebis classées à 2 pics.

En fait, les tableaux $4 b$ et 5 expriment la même chose, puisque les brebis qui cèdent rapidement leur lait sont probablement des brebis à I pic et que ces dernières ont une production plus faible mais un volume d'égouttage plus important (LABUsSIÈRE, I 967$)$.

TABLEAU 5

Vitesse de traite (essai en station)

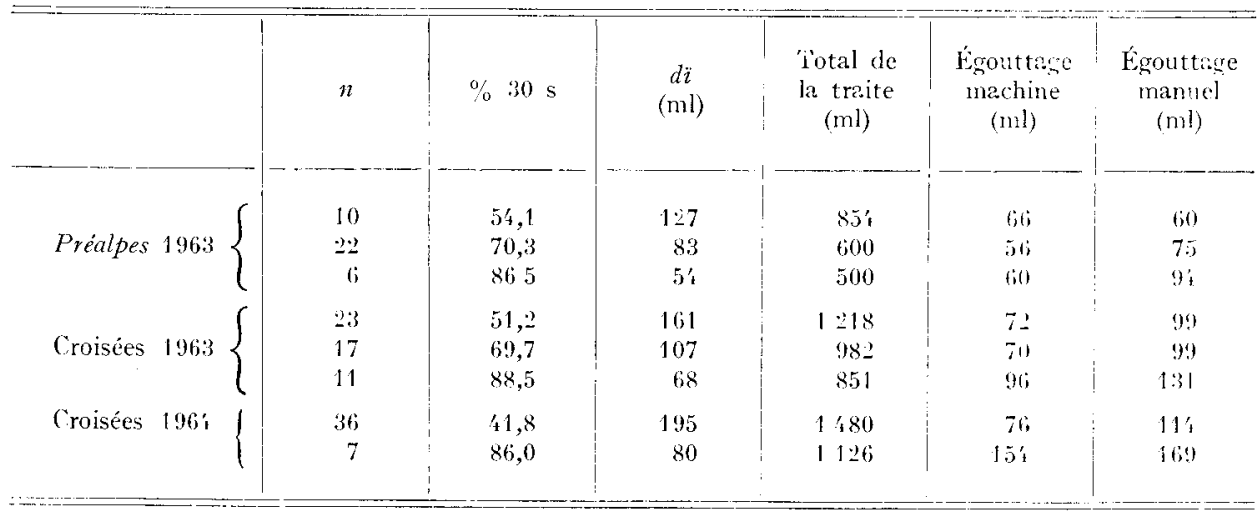

En I963, la descente de lait avait été répétée à 20 jours d'intervalle, en fin de période $I$ et de période 2 . La suppression de l'égouttage n'a pratiquement pas modifié l'écoulement du lait, mais cela demande à être vérifié par une technique plus fine que celle utilisée à l'époque.

\section{IV. — Répétabilité et différences entre béliers}

Pour les 40 brebis croisées, contrôlées 2 années de suite dans les mêmes conditions, le coefficient de corrélation entre années de la variable $d_{i}$ est significatif mais faible $(r=0,39)$. L'analyse de la variance des $d_{i}$ révèle une différence significative entre béliers malgré le petit nombre d'observations ( 7 pères et 89 produits). La chute de production consécutive à la suppression de l'égouttage est donc un caractère assez peu répétable mais certainement héritable.

\section{DISCUSSION}

I. Chez les Bovins, la suppression de l'égouttage manuel entraîne une chute de production relativement faible lorsque le volume de l'égouttage est lui-même peu élevé : 2 à 3 p. Ioo de perte en moyenne pour WIISON et CANNON (I934), WOODWARD, 
Ho'Ts et Graves (I936), pour un volume d'égonttage d'environ 5 p. I0o; 3 à I2 p. IOo de perte pour BAIIEY et al. (I953) pour un volume d'égouttage de IO p. IOO. EsskEAI. (I95I) puis HAxsEx (I963) ont également obtenu de très bons résultats au Danemark en remplaçant la finition à la main par une finition à la machine. I'après des observations effectuées sur génisses, CroUgh (I964) constate que la perte de production consécutive à la suppression de l'égouttage-machine n'est pas supérieure à la quantité de lait laissé dans la mamelle, c'est-à-dire $270 \mathrm{~g}$ de lait par jour et par traite. Plus récemment GoFf et SchMidT (I967) ont même montré que la suppression de 1'égouttage-machine sur des vaches de races Ayrshire, Jersiaise ou Brune des Alpes n'avait aucune influence significative sur le niveau de production moyen (I 9,09 contre I 9, I $3 \mathrm{~kg}$ de lait par jour), les différences individuelles étant en moyenne plus faibles pour les animaux à haut niveau de production.

2. Wans cette étude consacrée aux brebis laitières, l'absence d'égouttage manuel se traduit par une réduction de production de 16 p. Ioo. L'essai n'ayant duré que 20 jours, on peut évidemment se demander quelle aurait été la réduction de production s'il avait été poursuivi pendant toute la période de traite. Cependant, tant après la suppression de l'égouttage (début de période 2) qu'après la reprise de cet égouttage (début période 3), la phase d'adaptation des brebis n'a pas duré plus de 5 jours (fig. 2). Dans une expérience portant sur toute la période de traite, Ricordeau, MARTINET et DENAMUR (I963) avaient obtenu une perte moyenne journalière de I55 $\mathrm{ml}$ à peu près constante, soit au total $23 \mathrm{p}$. Ioo du témoin. En I967, BoSc, FisMANT et RICORDEAU enregistrent une perte de I I,8 p. IOo (à durée de traite égale) chez les Lacame adultes, de 6,8 p. roo pour les agnelles Lacaune et seulement de 3,7 p. Ioo pour les brebis Sardes.

I)ans toutes ces études et pour toutes les brebis adlultes Préalpes ou Lacanne, la chute de production consécutive à la suppression de l'égouttage manuel est supérieure au volume d'égouttage observé dans les lots témoins. Elle est légèrement plus importante pour les brebis à haut niveau d'égouttage mais plus faible qu'on ne pouvait l'espérer du fait que, pendant la période expérimentale, ces brebis ont tendance à mieux céder leur lait à la machine que les brebis à bas niveau d'égouttage.

I a perte de production est en moyenne plus importante pour les brebis à haut niveau de production, mais on sait par ailleurs (RICORDEAU et DENAMUR, I962) que la régression de production laitière avec le stade de lactation est en corrélation positive avec le niveau de production. Si l'on tient compte de cette régression, en calculant la différence entre la production totale moyenne des périodes I et 3 et celle de la période 2 ( $\mathrm{D}_{i}$ corrigée, tabl. 2$)$, on constate que l'effet du niveau de production n'est plus significatif.

3. Fin fin de compte, le volume d'égouttage est intéressant à contrôler pour obtenir une estimation correcte des performances laitières réellement obtenues dans les conditions habituelles de traite, mais il semble inutile de le retenir comme critère de sélection. Si l'on veut améliorer le niveau de production et réduire l'importance relative du volume de lait obtenu à l'égouttage manuel, il faut sélectionner les animaux sur les performances qu'ils manifestent lors de la traite à la machine.

4. Lans l'immédiat, on ne peut supprimer l'égouttage manuel qui représente une part importante de la traite, mais on peut le remplacer par une deuxième pose des gobelets puisque cette solution donne des résultats aussi bons avec les brebis 
(Bosc, Flamant et Ricordeau, ig67) qu'avec les bovins laitiers (Politiek et Rooy, I962). On peut aussi chercher à améliorer sérieusement la préparation de la mamelle avant la traite (comme dans l'espèce bovine) et envisager des croisements avec des races faciles à traire (la race Sarde par exemple) pour obtenir des animaux capables de donner tout leur lait à la machine.

Reçu pour publication en mars 1968.

\section{REMERCIEMENTS}

L'essai en ferme a été subventionné par un fonds de concours Mouton (F. N. O.). La réalisation pratique des contrôles est due à M. Castanier du G. R. C. E. 'T. A., à MM. ÉTIEnNE et Carpentier techniciens I. N. R. A. et à M. Peyraud, stagiaire I.'T. P. A. Nous remercions tout spécialement M. Delmas du G. R. C. E. T. A. du sud de l'Aveyron qui a assuré la mise en place de l'essai et sa bonne réalisation.

L'essai en station a été réalisé avec la collaboration technique de MM. Petrequin et Mirman.

\section{SUMMARY}

MACHINE MILKING OF THE EWE. CONSEQUENCES OF THE SUPPRESSION OF HAND STRIPPING DEPENDING UPON MILKING CHARACTERISTICS

The current study was undertaken to estimate the variations in milk yield induced by the suppression of hand stripping, depending upon the amounts of milk obtained during the various stages of milking. Two experiments were made : one in a farm on 202 Lacaune ewes (with ror control) milked after a $3^{8}$ day suckling period; one in a research centre on $3^{8}$ Préalpes $d u$ Sud and 5 I crossbred ewes milked since lambing. From the 5 oth day of lactation on wards three periods were considered : period I : (ro days) : machine milking + hand stripping; period 2 (2o days) : machine milking without stripping; period 3 (10 days) : machine milking + hand stripping again.

In the farm experiment, the suppression of hand stripping induces a ir $8 \mathrm{ml}$ daily loss ( 6 percent) superior to the volume $(93 \mathrm{ml})$ hand stripped in the control ewes (table I, fig. I and 2 ).

The total difference in milk yield between periods $\mathrm{I}$ and 2 (total $\mathrm{D}_{i}$ or $\mathrm{D}_{i}$ adjusted for the level of production) is in a positive correlation $(r=0.30$ and 034 , table 2$)$ with the volume of stripping obtained in period I. On the contrary, the deviation between milk quantities obtained before and after suppression of stripping $\left(\mathrm{d}_{i}\right)$ significantly varies (tables 3 and 4 ) in inverse ratio to the volume of stripping and seems lower with ewes giving the greater part of their milk for the first 30 seconds of milking (table 5).

From a genetic viewpoint, to improve the production level and reduce the comparative amount of hand-stripped milk, the ewes must not be selected on the criterion of total milking performances, but on the amount of milk obtained by machine milking exclusively.

\section{RÉFÉRENCES BIBLIOGRAPHIQUES}

Bayley G. L., Clolgh P. A., Doon F. H., Boot A. S., Rowland S. J., I953. The effect of incomplete milking on the secretion of milk. XIII Int. Dairy Congr., vol. 2. 76-S2.

Bosc J., 1963. Problemes de l'organisation du travail dans un troupeau de brebis laitières. Adaptation de la méthode arête de poisson à la traite mécanique des brebis. Mécanisation et Automation en Élevage. A. F. Z., Paris, 39 pp.

Bosc J., Flamant J. C., Ricordeau G., ig67. Traite à la machine des brebis. Suppression de l'égouttage manuel ou remplacement par un égouttage machine. Ann. Zootech., 16, I92-202.

Crotgu P. A., I964. Machine stripping, is it really necessary? Agriculture, Lond., 71, $3^{61-363 .}$ 
Eskedal H. W., 195г. Quelques expériences sur la traite des vaches (en danois). Beretn. Forsögslab., 255, 34-60.

Goff K. R., Schuid' $\quad$ G. H., 1967. Effect of eliminating machine stripping of dairy cows on milk production, residual milk and mastitis. J. Dairy Sci., 50, г $787-1791$.

HANSEN H. E., I 963 . Comparaisons of machine milking with and without hand stripping. Alfa Laval symposium on machine milking. Hamra, $146-\mathrm{I} 47$.

LABUSsiÉre J., 1967. Relations entre le niveau de production laitic̀re des brebis et leur aptitude à la traite, $X V I I^{\mathrm{e}}$ Congr. int. Laiterie, Munich. Section $A 1,43$ à 51.

Labussiḱre J., Martinet J., ig64. Description de 2 appareils permettant le contrôle automatique des débits de lait au cours de la traite à la machine. Premiers résultats obtenus chez la Brebis. Ann. Zoolech., 13, 199-212.

Politiek R. D., Rooy J. de, 1962. Hand or machine stripping? Ned. Melk en Zuivelijdschr., 17, I 54. (Abstract).

Ricorneau G., Denamur R., ig62. Production laitière des brebis Préalpes du Sul pendant les phases d'allaitement, de sevrage et de traite. Ann. Zootech., 11, 5-38.

Ricordeau G., Martinet J., Denamur R., ig63. Traite à la machine des brebis Préalpes du Sud. Importance des différentes opérations de la traitre. Ann. Zootech., 12, 203-225.

Wilson J. L., Cannon C. Y., I934. The value of hand stripping after machine milking. J. Dairy Sci., $17,33 \mathrm{I}-33^{8}$.

Woodward T. E., Hotis R. P., Graves R. R., 1936. Incomplete milking in relation to milk production. Tech. Bull. U.S.Dep., Agric., 522. 